\title{
Erratum: Sudden onset of mortality within a colony of $\mathrm{FVB} / \mathrm{n}$ mice
}

Matthew D. Rosenbaum, DVM, Sue VandeWoude, DVM \& Helle Bielefeldt-Ohmann, DVM, PhD

Lab Anim. (NY) 36, 15-16 (2007).

The sections shown in Figure 2 are of the hippocampus, not of the hypothalamus. 


\title{
Sudden onset of mortality within a colony of FVB/n mice
}

\author{
Matthew D. Rosenbaum, DVM, Sue VandeWoude, DVM \& Helle Bielefeldt-Ohmann, DVM, PhD
}

An investigator at Colorado State University received five breeding pairs of $\mathrm{FVB} / \mathrm{n}$ mice less than 23 days old from a commercial vendor. We housed the mice in a semibarrier animal room in microisolator cages on a 12-h light/dark cycle and gave them ad libitum access to water and rodent chow (Harlan Teklad 8640, Madison, WI). Serologic evaluations of two sentinel ICR mice caged in the same room were negative for serum antibodies reacting with mouse hepatitis virus (MHV), minute virus of mice (MVM), Sendai virus, mouse parvovirus (MPV), Mycoplasma pulmonis, Theiler's murine encephalomyelitis virus (TMEV), and epizootic diarrhea of infant mice (EDIM) antigens. One mouse tested negative for all of the aforementioned except for MPV. The five pairs of FVB/n mice were therefore moved to a different MPV-positive semi-barrier room that also housed immunocompromised strains of mice.

Three of the five females subsequently gave birth to $37 \mathrm{FVB} / \mathrm{n}$ offspring ( 17 males and 20 females) over a four-day period. Animal care technicians weaned the pups at 21 days old. Four weeks later, they noticed wounds on the tails and tail bases of 7 week-old female FVB/n mice in two cages. One of us (M.D.R.) diagnosed conspecific aggression as the likely etiology and separated the animals in an attempt to alleviate the intracage aggression. We did not note any other abnormalities in the $\mathrm{FVB} / \mathrm{n}$ mice or in any of the other mice housed in the same room.

Approximately 5 weeks later (when the mice were 12 weeks old), we found a dead female FVB/n mouse with no premonitory symptoms. M.D.R. performed a complete necropsy; no gross lesions were noted. Over
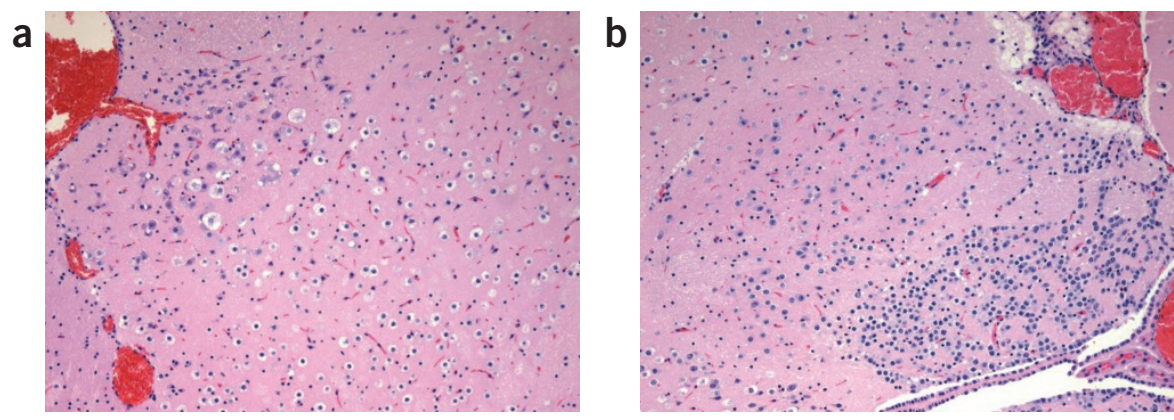

FIGURE 1 | Photomicrographs of brain sections taken from (a) a 14-week-old female FVB/n mouse found dead with nonspecific gross lesions and no premonitory signs and (b) a clinically normal 14-week-old $\mathrm{FVB} / \mathrm{n}$ mouse. The sections are of the thalamus. In the brain of the mouse that died suddenly (a), many of the neurons are undergoing degeneration and necrosis, most likely due to acute ischemia. (H\&E stain; low-medium (a) and low (b) power magnification.)

the next 4 weeks, animal care technicians found an additional 16 mice ( 1 male, 15 female) dead in their cages, again without any premonitory symptoms.

We took blood from one of the remaining five female mice and submitted serum for a serology panel that tested for antibodies to ectromelia virus, EDIM, lymphocytic choriomeningitis virus (LCM), M. pulmonis, MHV, MNV, Parvo NS-1, MPV, MVM, pneumonia virus of mice (PVM), RE03, Sendai, and TMEV-GDII; all test results were negative. M.D.R. performed necropsies on 10 of the mice that had previously been found dead ( 9 female, 1 male). All mice were considered in good body condition, with a score of $>3$ out of 5 (ref. 1). The mice had adequate body fat stores and food within the stomach; the small and large intestines contained normal amounts of ingesta and feces. All mice appeared normally hydrated. We noted occasional superficial excoriations of the dermis on the dorsum of the tail base. Additionally, all the mice had wet fur below the mandible and on the ventrum of the neck.

We performed histopathologic examinations on all major organs (brain, liver, heart, lungs, stomach, spleen, lymph nodes, salivary glands, small intestine, colon, kidney, adrenals, bladder, and gonads) from four mice that were necropsied. Brain sections of all mice had multifocal areas of neuronal necrosis and neuronal depletion in the cerebral cortex (Fig. 1). We also detected astrocytosis and early gliosis in the brain of one of two affected mice that were labeled with an antibody that recognizes glial fibrillary acidic protein (GFAP), a cell marker for neuroglia.

Are these brain lesions of neuronal necrosis and loss common among $\mathrm{FVB} / \mathrm{n}$ mice? Does the case history and epidemiology point to a syndrome associated with this strain?

\section{What's your diagnosis?}




\section{Diagnosis | 'Space cadet' syndrome of female FVB/n mice}

At a 1999 NIH Symposium on transgenic mice, Deborah E. Devor-Hennemann reported a syndrome of sudden death and variable CNS lesions in both transgenic and wildtype populations of FVB mice and named it 'space cadet' syndrome (SCS) ${ }^{2}$. The behavioral manifestations of this syndrome, including withdrawal from social interaction, led to the unique and memorable name. A previous report has shown that while both sexes are affected, females are more predisposed to $\mathrm{SCS}^{3}$. Some investigators have defined this condition as a neuroendocrinological disorder that may be the result of a mutation first reported in the FVB/NCr subline and lines derived from $\mathrm{NCr}$ stock ${ }^{2}$. Others hypothesize that this syndrome represents a potentially longstanding condition of FVB mice that had not been previously reported ${ }^{2}$.SCS is thought to be the result of neuronal necrosis in the brain from seizure activity and its associated hypoxemia, leading to the behavioral changes in survivors ${ }^{4}$. Histologically, these degenerative brain lesions are characterized by neuronal degeneration and necrosis (Fig. 1a), predominantly seen in the thalamus, but also appearing in the cerebral cortex and hippocampus. Concurrent astrocyte hypertrophy (or astrocytosis) in the areas of neuronal necrosis, as evidenced by an increase in glial fibrillary acidic protein (GFAP) staining (Fig. 2a), results eventually in gliosis (the production of a dense fibrous network of neuroglia).

SCS has been linked to reduced fertility, aggression toward cagemates, and excessive postpartum infanticide. This condition should be considered as a differential diagnosis in the likely causes of death in otherwise clinically healthy female FVB/n mice ${ }^{3}$. SCS should also be a differential diagnosis for either abrupt behavioral changes among littermates or an unexpected change in breeding production within a FVB colony of mice.

Other clinical signs reported in SCS include seizures (which may be fatal) and hyper-reactivity to stimuli. Seizures are the most probable cause of death in the mice in this report, as this is consistent with the lack of gross lesions in most of the animals. The presence of saliva on the ventrum of the neck in the animals described here also supports
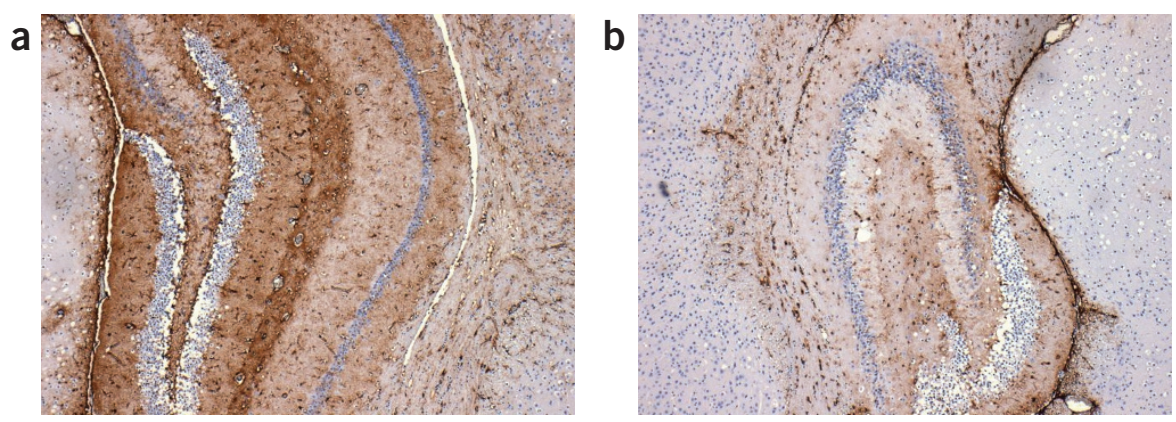

FIGURE 2 | Photomicrographs of the brain taken from two 14-week-old female FVB/n mice found dead with nonspecific gross lesions and no premonitory signs. Both sections are of the hypothalamus. Glial fibrillary acidic protein (GFAP) antibody has been used as an immunostain for glial cells, which stain strongly brown. In (a), there is increased labeling for the immunostain (GFAP) most likely from astrocytes filling in the areas of neuronal dropout. It is likely this animal had multiple seizure episodes prior to death that caused ischemic neuronal necrosis and subsequent dropout of neurons. The histopathological appearance of the brain in this mouse is consistent with lesions associated with status epilepticus in humans. In contrast, (b) shows immunohistochemical labeling for GFAP within the normal range, indicative of a normal number of neuroglia and suggesting that this animal most likely experienced a single acute and fatal epileptic episode. (Hematoxylin counterstain; low-medium (a) and low (b) power magnification.) the possibility of an episode of convulsing or overactive chewing just prior to death. There has been no definite link to external stimuli resulting in increased seizure activity, though the association of the syndrome in these mice with movement to different rooms within the facility is an intriguing observation that might suggest an etiology for increase in audiogenic seizure activity.

Gross pathological changes associated with SCS that were not seen in the mice reported here include distention of the urinary and gall bladders, adrenal hypertrophy, and brain hypertrophy, possibly secondary to widespread gliosis ${ }^{2}$. Histopathological findings noted in SCS include laminar necrosis of cerebral cortical and hippocampal neurons accompanied by poliomalacia and gliosis ${ }^{2}$. Clinical signs reported in SCS but not noted in these mice include polyuria and polydipsia that may be of neuroendocrine origin ${ }^{2}$.

This report emphasizes that background genotype must be considered during knockout and transgenic phenotype analysis. This observation was realized over a decade ago by behavioral neuroscientists; consequently, investigators in this field recommend that knockout mutations be maintained as inbred congenic lines or as $\mathrm{F}_{1}$ hybrids between two strains. This allows accurate analysis of the phenotype attributable to the transgene alone ${ }^{5}$.

1. Ullman-Cullere, M.H. \& Foltz, C.J. Body condition scoring: a rapid and accurate method for assessing health status in mice. Lab. Anim. Sci. 49(3), 319-323 (1999).

2. Ward, J.M., Anver, M.R., Mahler, J.F. \& DevorHennemann, D.E. in Pathology of Genetically Engineered Mice (eds. Ward, J.M., Mahler, J.F., Maronpot, R.R. \& Sundberg, J.P.) 161-179 (Iowa State University Press, Ames, IA, 2000).

3. Goelz, M.F. et al. Neuropathologic findings associated with seizures in FVB mice. Lab. Anim. Sci. 48(1), 34-37 (1998).

4. Auerbach, A.B. et al. Strain-dependent differences in the efficiency of transgenic mouse production. Transgenic Res. 12(1), 59-69 (2003).

5. Silva, A.J. et al. Mutant mice and neuroscience: recommendations concerning genetic background. Banbury Conference on genetic background in mice. Neuron 19(4), 755-759 (1997). 